\title{
Bruxismo do sono e sua associação com distúrbios do sono em policiais
}

\section{Sleep bruxism and its associations with sleep disorders in policemen}

\author{
Jeanne Paiva de Siqueira COELHO \\ Sílvia Carneiro de LUCENA \\ Cirurgião-Dentista - UFMA - Universidade Federal do Maranhão - São Luis - Brasil
}

\author{
Andréa Lúcia Almeida de CARVALHO \\ Professora Doutora de Prótese Dental - UFMA - Universidade Federal do Maranhão - São Luis - Brasil
}

Fernanda Ferreira LOPES

Professora Doutora de Semiologia - UFMA - Universidade Federal do Maranhão - São Luis - Brasil

\author{
Ana Emilia Figueiredo de OLIVEIRA \\ Professora Doutora de Radiologia - UFMA - Universidade Federal do Maranhão - São Luis - Brasil
}

\begin{abstract}
Resumo
O bruxismo do sono é uma das atividades parafuncionais mais abordados na Odontologia, especialmente devido a questões controversas quanto à sua prevalência e etiologia. Os estudos realizados na área conseguem, tão somente, amenizar seu potencial destrutivo, mas não estabelecem, todavia, uma relação direta entre causa e efeito. A literatura considera atualmente o estresse, a fisiologia do sono, algumas drogas e, em menor escala, a oclusão, como fatores desencadeadores deste comportamento parafuncional. O objetivo deste trabalho foi investigar a relação entre bruxismo do sono e distúrbios do sono, em policiais militares da cidade de São Luís-MA. Os resultados revelaram que não houve associação significante entre bruxismo do sono e distúrbios do sono $(\mathrm{p}=0,07)$. Conclui-se, portanto que apesar de o bruxismo ocorrer durante o sono, ele não interfere na qualidade do mesmo.
\end{abstract}

\section{UNITERMOS}

Bruxismo; bruxismo do sono; transtorno do sono; policia.

\section{INTRODUÇÃO}

O bruxismo tem sido um dos assuntos mais discutidos na Odontologia, principalmente em razão de seu potencial destrutivo e ainda por ser mal compreendido nos seus fatores etiológicos. Mesmo com o avanço dos estudos acerca do tema, ainda não se consegue evitar ou paralisar o bruxismo, mas somente minimizar seus efeitos deletérios. Entende-se que alguns fatores diminuem sua intensidade, enquanto outros a aumentam. Entretanto, a multifatoriedade dificulta a compreensão e o controle desta parafunção.
De acordo com a Classificação Internacional de Distúrbios do Sono, o Bruxismo do Sono (BS) é uma atividade oral caracterizada pelo ranger ou apertar dos dentes durante o sono geralmente associado à microdespertares, o BS passou a ser nomeado de bruxismo relacionado ao sono e foi caracterizado como distúrbio de movimento relacionado ao sono ${ }^{17}$. $\mathrm{O}$ bruxismo pode ocorrer tanto em vigília quanto durante o sono. Considerando-se que a vigília e o sono são estados fisiológicos bem distintos, o bruxismo durante a vigília deve ser considerado uma entidade separada do bruxismo do sono. 
O BS tem sido confirmado como um distúrbio de movimento relacionado ao sono; porém também se acredita que ele está relacionado a fatores psicossociais e que o estresse é um fator iniciante, predisponente e perpetuante do bruxismo ${ }^{25}$. Segundo Barder e Lavigne $^{3}$ (2000), a correlação entre bruxismo do sono e fatores emocionais tem sido freqüentemente relatada na literatura. Um estudo realizado por Kampe et al $^{16}$ (1997) revelou que pacientes bruxistas estavam mais predispostos à ansiedade e mais vulneráveis a desordens psicossomáticas. Outros elementos endógenos podem influenciar no bruxismo, como os fatores genéticos, os neuroquímicos, as desordens neurológicas e psiquiátricas e os distúrbios do sono como: movimento periódico das pernas - PLMS, a apnéia e os distúrbios do comportamento REM ${ }^{17}$.

O sono normal possui uma arquitetura claramente definida: estágios 1, 2, 3 e 4 de sono não REM (Rapid Eye Movements- rápido movimentos dos olhos), seguidos pelo sono REM. Durante o tempo total de sono, normalmente ocorrem de 4 a 6 ciclos bifásicos com duração de 90 a 100 minutos. Cada ciclo é composto pelas fases de sono não REM, com duração de 45 a 85 minutos, e pela fase de sono REM, que dura de 5 a 45 minutos ${ }^{10}$. Os episódios de bruxismo ocorrem principalmente durante os estágios 1 e 2 do sono não REM e estão associados a episódios de microdespertar ${ }^{9}$.

Clarke, Townsend ${ }^{9}$ (1984) sugerem ainda que os movimentos do corpo ocorram durante o sono REM devido aos impulsos do sistema nervoso central. Caso o bruxismo ocorra também nessa fase do sono, suportaria o conceito da etiologia de uma origem central. Tal associação torna improvável que fatores patogenéticos exógenos e periféricos, como as interferências oclusais forneçam o estímulo para o bruxismo do sono ${ }^{8}$. Acredita-se ainda, que não bastaria a presença de bruxismo durante o sono para causar sintomatologia dolorosa no sistema mastigatório, mas seu padrão e relacionamento com os estágios do sono eram os fatores que afetariam os sintomas clínicos ${ }^{26}$. Rugh, Harlan ${ }^{22}$ (1988) afirmaram que, embora os episódios de BS pareçam ocorrer no estágio 2 do sono não REM e durante atividades de microdespertar, os episódios que ocorrem durante o sono REM podem ser mais danosos as estruturas orais.

Apesar do BS ser há muito enquadrado como uma desordem de sono, e ser considerada como a terceira mais freqüente, os conhecimentos referentes a sua natureza e tratamento estão aquém daqueles referentes a outras desordens como, por exemplo, a síndrome da apnéia obstrutiva do sono ${ }^{17}$.
Saletu et al. ${ }^{23}$ (2005) investigaram os efeitos agudos de Clonazepam comparados com placebos utilizando polissonografias e psicometria em 10 pacientes com BS. A qualidade de sono foi avaliada de forma objetiva determinada por polissonografia, e subjetiva determinada por seis escalas: Escala Zung para automedição de depressão e de Ansiedade, Escala de Sono de Epworth, Escala de Avaliação Internacional de Síndrome de Pernas Inquietas, Índice de Qualidade de Vida de acordo com Mezzich e Cohen e Índice de Qualidade de Sono de Pittsburgh. Os resultados mostraram que a terapia aguda com Clonazepam melhorou significativamente não só o índice de bruxismo, mas também a qualidade de sono objetiva e subjetiva.

O diagnóstico clínico do bruxismo do sono é complexo, não apenas porque o aparecimento dos sinais e sintomas varia ao longo do tempo, como também pela não confiabilidade dos relatos de ranger de dentes. Apesar da difícil operacionalização, existem muitos estudos que avaliam, através de questionários, possíveis interações entre bruxismo e seus fatores etiológicos. Como a credibilidade desses estudos foi sempre questionada, em 1996 Lavigne et al. ${ }^{18}$ testaram a validade clínica dos critérios de diagnóstico para o bruxismo através de um estudo comparativo dos resultados de registros polissonográficos avaliados sem o conhecimento do diagnóstico clínico de bruxismo. A comparação destes mostrou $81,3 \%$ de especificidade (percentagem de pacientes controle sem bruxismo) e $83,3 \%$ de sensibilidade (percentagem de pacientes com bruxismo). concluindo que o diagnóstico clínico de bruxismo foi correto em $81,3 \%$ dos pacientes controle e $83,3 \%$ dos bruxistas.

$\mathrm{O}$ fato de o bruxismo ocorrer durante o sono gera incerteza quanto à interferência deste comportamento parafuncional na qualidade do sono. Não se discute a supremacia do exame polissonográfico para o diagnóstico de BS, mas o alto custo e a falta de comodidade para o paciente durante o exame são fatores que devem ser levados em consideração. Assim, o objetivo desta pesquisa foi verificar a relação entre Bruxismo do sono, diagnosticado através de questionários e exame clínico, e distúrbios do sono determinadas pelo Índice de Qualidade de Sono de Pittsburgh.

\section{Material e Método}

Após aprovação pela Comissão Nacional de Ética em Pesquisa do Hospital Universitário Presidente Dutra- UFMA ( ${ }^{\circ}$ 33104-0429/2004), 643 homens pertencentes a Policia Militar de São Luis - MA 
foram examinados. O critério de inclusão foi apresentar boa saúde e ter pelo menos 26 dentes ${ }^{15,16}$. Foram excluídos indivíduos com ausência de suporte dental posterior, que usavam próteses dentárias, que tivessem feito algum tratamento irreversível para Disfunção Temporo-mandibular ou ajuste oclusal, com desordens neurológicas ou psiquiátricas, que faziam uso excessivo de álcool ou drogas e de medicamentos com ação no sistema nervoso central ou com presença de maloclusão grave ${ }^{18}$. Dessa população, apenas 90 pacientes, com idade entre 22 e 45 anos, foram selecionados e estes foram submetidos a anamnese e exame clínico.

Durante a anamnese e exame físico, sinais e sintomas de bruxismo foram investigados tais como fadiga nos músculos mastigatórios ao despertar, história de ruídos de ranger de dentes durante o sono (nos últimos seis meses), confirmada por um companheiro de quarto; relato de musculatura mandibular rígida ou fatigada durante a noite e/ou ao despertar; sensibilidade dolorosa à palpação nos músculos masseter e/ ou temporal; e hipertrofia dos músculos masseteres. O exame clínico também investigou a presença de hipertrofia do músculo masseter e a sensibilidade à palpação do masseter e temporal ${ }^{11}$. O exame intra-oral avaliou a presença de facetas de desgaste em esmalte e dentina através da inspeção visual das faces incisal e oclusal dos dentes anteriores e posteriores, com auxilio de um espelho bucal ${ }^{2,14,22}$. Então, as facetas de bruxismo foram distinguidas das causadas pela atrição fisiológica durante a mastigação. Para isso, solicitouse ao pacientes que realizasse movimentos protusivos e laterais com a mandibular para verificar o possível alinhamento das facetas em dentes antagonistas posto que a mastigação normal reflete padrão de desgaste caracterizado por facetas em dentes antagônicos que não se alinham enquanto que as facetas de desgaste apresentadas por pacientes bruxistas caracterizam-se pelo alinhamento com dentes antagonistas ${ }^{2,22}$.
O diagnóstico de bruxismo do sono foi uma adaptação dos critérios adotados por Rugh e Harlan ${ }^{22}$ (1988); Lavigne et al. ${ }^{18}$ (1996), Carvalho et al. ${ }^{7}$ (2008). Foram considerados bruxistas os pacientes com presença de facetas incisais ou oclusais alinhadas com desgaste em grau 1, 2 ou 3 (incompatíveis com idade e função), associada à presença de pelo menos um dos seguintes sinais e sintomas: história de ruídos de rangimento durante o sono (nos últimos seis meses), confirmada por um companheiro de quarto; relato de musculatura mandibular rígida ou fatigada durante a noite e/ou ao despertar; sensibilidade dolorosa à palpação nos músculos masseter e/ou temporal e hipertrofia dos músculos masseteres.

A presença de distúrbios do sono foi investigada por meio do Índice de Qualidade de Sono de Pittsburgh (IQSP), que se constitui em 18 itens de auto-avaliação da qualidade geral de sono, provendo informações sobre o número de horas efetivas de sono, número de perturbações de sono, latência do sono, eficiência do sono e uso de medicamento para dormir. O IQSP é um instrumento seguro para avaliar a qualidade global de sono e possíveis perturbações, com sensibilidade de $89,6 \%$ e especificidade de $86,5 \%$. Pontuações maiores que 5 no questionário sugerem a ocorrência de distúrbios do sono ${ }^{5,6}$.

Para verificar a presença de associação entre o bruxismo do sono e o distúrbio do sono foi aplicado o teste não-paramêtrico do qui-quadrado, com nível de significância de $5 \%$.

\section{Resultados}

A prevalência de BS nos 90 policiais militares foi de $31,11 \%$ e a prevalência de distúrbios do sono foi de $53,4 \%$ (Tabelas 1 ).

Tabela 1 - Prevalência de Bruxismo do sono (BS) e de Distúrbio do sono (DS) entre os policiais
militares (São Luís - MA)


Foi realizada a distribuição percentual de BS em policiais com e sem distúrbios do sono. Para isso, a amostra total de policiais selecionados (90) foi dividida em dois grupos: grupo A contendo apenas os policiais sem distúrbios do sono $(n=42)$ e grupo $B$ contendo apenas os policias com distúrbios do sono $(n=48)$. Os resultados indicaram que, no grupo de pacientes sem distúrbios do sono, a presença de BS foi de $20,9 \%$ enquanto que, no grupo de pacientes com distúrbios do sono, o percentual de pacientes com BS elevou-se para $40,4 \%$.

Do mesmo modo, foi realizada também a distribuição percentual de distúrbios do sono em policiais com BS e policiais sem BS. Dividindo o total de pacientes (90) em dois grupos: grupo A contendo apenas os policiais sem BS $(n=62)$ e grupo B con- tendo os policiais com BS $(\mathrm{n}=28)$. Os resultados obtidos indicaram que no grupo de pacientes sem BS, 45,2\% apresentaram distúrbios do sono enquanto que, no grupo de pacientes com BS, o percentual de pacientes que apresentavam distúrbios do sono elevou-se para $67,9 \%$.

A frequiência relativa de BS e de distúrbios do sono em 90 policiais militares revelou que, $36,78 \%$ dos policiais não apresentaram nem BS nem distúrbios do sono; $31,10 \%$ apresentaram somente distúrbio de sono; $10 \%$ apresentaram somente BS e $21,10 \%$ dos policiais apresentaram BS e distúrbios do sono (Gráfico 1).

Ao avaliar os dados obtidos utilizando o teste não paramétrico do Qui quadrado com correção de Yates, não foi encontrada relação significante entre BS e distúrbio do sono $(\mathrm{p}=0,07)$.

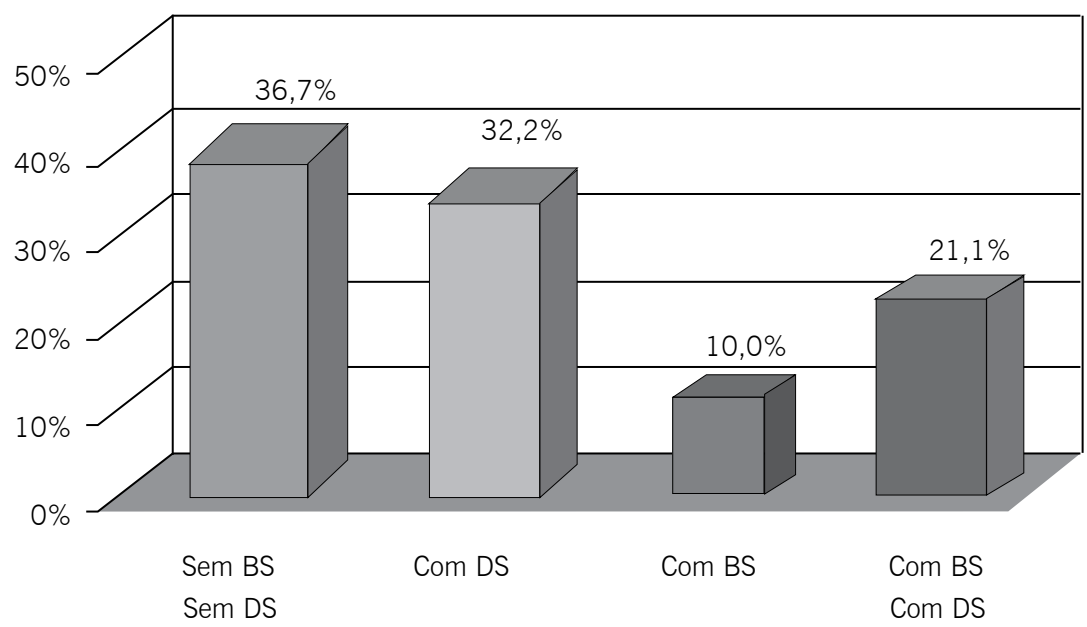

Gráfico 1. Distribuição de bruxismo do sono (BS) e distúrbios do sono (DS) em 90 policiais militares. 


\section{Dıscussão}

O diagnóstico de bruxismo, em geral, é realizado primeiramente por meio do exame físico (anamnese e exame clínico). Porém, a literatura nos mostra que este pode ser feito através de diversos métodos, como avaliação de desgaste dental em modelos de estudo, aplicação de questionários, eletromiografias e polissonografias em laboratório do sono. $\mathrm{O}$ uso isolado de qualquer um destes instrumentos de diagnóstico pode causar dificuldades na definição da prevalência real de bruxismo, subestimando ou superestimando os resultados.

A prevalência de bruxismo do sono neste estudo foi de $31,11 \%$. Esses resultados diferem do trabalho de Carvalho et al. ${ }^{7}$ (2008), que encontrou uma prevalência de $50,25 \%$ de bruxismo em policiais militares da cidade de Campinas - SP e diferem, também, dos resultados de Rodrigues et al..$^{21}$ (2001) que encontraram uma prevalência de $74,4 \%$. O primeiro trabalho, entretanto, considera bruxismo durante a vigília e bruxismo do sono, enquanto o último foi realizado em grupos de pacientes com DTM, o que compromete seus resultados, pois segundo Ciancaglini et al. ${ }^{8}$ (2001), em pacientes com DTM é encontrada maior frequiência de bruxismo. Por outro lado, os presentes resultados foram semelhantes aos encontrados por Glaros ${ }^{12}$ (1981) que, após pesquisar 1052 pacientes, encontrou que $30,2 \%$ (232) eram bruxistas. Prevalência inferior $(8,2 \%)$ foi encontrada por Ohayon et al. ${ }^{20}$ (2001) em pesquisa realizada com 13.057 indivíduos com faixa etária entre 15 e 100 anos de idade. Acredita-se, no entanto, que este resultado pode estar subestimado, uma vez que obtido somente através de auto-relato sobre o ranger de dentes, que é um hábito inconsciente e de difícil confirmação pelo companheiro de quarto. Outros estudiosos, como Selligman et al. ${ }^{24}$ (1988) e Attanasio ${ }^{2}$ (1977), encontraram prevalências superiores com valores acima de $90 \%$, que podem estar superestimadas, pois se basearam apenas na análise de facetas de desgaste. Estas têm etiologia multifatorial, e seu desgaste pode ser acentuado por outros fatores que não o bruxismo, tais como substancias ácidas, escovação, apreensão de objetos com os dentes ou mesmo contatos durante a mastigação ${ }^{13}$.

Os resultados deste trabalho mostraram que não houve relação estatisticamente significante entre distúrbios do sono e bruxismo do sono $(\mathrm{p}=0,07)$. Esses resultados estão de acordo com os trabalhos de Macaluso et al. ${ }^{19}(1998)$ que afirmam que os pacientes com bruxismo do sono não apresentam diferenças significativas entre as variáveis convencionais do sono em relação a pacientes sem bruxismo; de Kato et al. ${ }^{17}$ (2001) e Lavigne ${ }^{18}$ (2001) que declararam que pacientes com BS têm uma macroestrutura de sono normal e geralmente não reclamam de distúrbios do sono; e com Watanabe et al ${ }^{27}$. (2003) que não encontrou relação significativa entre bruxismo e as variáveis, dor de cabeça e perturbações de sono, em seu estudo que visou correlacionar bruxismo do sono a comportamentos diários (estresse, atividade física) e qualidade do sono.

O fato de BS estar associado a eventos de microdespertar não significa que a qualidade de sono subjetiva será alterada; além do que, a Associação Americana de Distúrbios do Sono, afirmou que episódios de microdespertar ocorrem cerca 14,7 vezes por hora de sono em um individuo adulto jovem normal ${ }^{17}$.

O presente resultado diverge dos resultados de Saletu et al. ${ }^{23}$ (2005) que encontraram escores acima de 5 no Índice de Qualidade de Sono de Pittsburgh em todos os pacientes diagnosticados com BS, ou seja, todos os pacientes bruxistas apresentavam distúrbio de sono; porém, esse trabalho foi realizado em uma amostra com idade média de 46,5 anos e, segundo Kato et al. ${ }^{17}$ (2001), idade maior que 40 anos é um fator que interfere na organização do sono. Os resultados deste estudo divergem também dos estudos de Bailey ${ }^{4}$ (1997), que afirma que a incidência de BS aumenta em pacientes com outras distúrbios do sono, especialmente apnéia do sono e mioclonus noturno.

Segundo Ahlbergh ${ }^{1}$ (2003), turnos irregulares de trabalho podem estar associados a problemas de saúde, a maioria destes ligados ao ciclo cicardiano. Além disso, horário irregular de trabalho foi apontado como uma das causas de distúrbios do sono, cansaço e estresse. O presente estudo revelou um alto índice de distúrbios do sono $(53,4)$ que podem ter como causa o turno irregular de trabalho do policial militar.

\section{Conclusão}

De acordo com os resultados obtidos neste estudo, pode-se concluir que, para esse grupo específico:

1) A prevalência de bruxismo do sono foi de $31,11 \%$

2) Não houve associação significante entre bruxismo do sono e distúrbios do sono avaliadas subjetivamente, portanto a atividade do bruxismo parece não interferir na qualidade do sono daqueles que o possuem. 


\section{Abstract}

Sleep bruxism is one of the most studied parafunctional activity in Odontology, specially because of the controversial aspects regarded to its prevalence and etiology. Studies in this field only show ways to decrease its destructive action, not showing, however, a straight relationship between cause and effect. The literature considers stress, sleep physiology, some drugs and, in a lower level, occlusion as starting factors for this parafunctional behavior. The aim of this paper was to assess the relation between sleep bruxism and sleep disorders. The results showed no relevant association between sleep bruxism and other sleep disorders $(\mathrm{p}=0,07)$. It is concluded that, despite occuring during sleep, bruxism events do not interfere in sleep quality.

\section{UNITERMS}

Bruxism; sleep bruxism; sleep disorders; police.

\section{Referencias}

1. Ahlberg K, Ahlberg J, Könönen M, Partinen M, Lindholm H, Savolainen A. Reported bruxism and stress experience in media personnel witch or without irregular shift work. Acta Odontol Scand 2003 Oct;61(5):315-8.

2. Attanasio R. An overview of bruxism and its management. Dent Clin North Am. 1997 Apr;41(2):229-1.

3. Bader G, Lavigne G. Sleep bruxism; an overview of an oromandibular sleep movement disorder. Sleep Med Rev 2000 Feb;4(1):27-43.

4. Bailey DR. Sleep disorders. Dent Clin North Am. 1997Apr;41(2): 189-209.

5. Beck SL, Schwartz AL, Towsley G, Dudley W, Barsevick A. Psycometric Evaluation of the Pittsburgh Sleep Quality Index in cancer patients. J Pain Symptom Manage. 2004 Feb;27(2):140-8.

6. Buysse DJ, Reynolds CF, Monk TH, Kupfer DJ. The Pittsburgh Sleep Quality Index: a new instrument for psychiatric practice and research. Psychiatry Res. 1989 May;28(2):193-213.

7. Carvalho ALA, Del Bel-Cury AA, Rodrigues-Garcia RCM. Prevalence and association of bruxism and emotional stress in Brazilian police officers. Braz Oral Res. 2008 Jan-Mar;22(1):31-5.

8. Ciancaglini R, Gherlone EF, Radaelli G. The relationship of bruxism with craniofacial pain and symptoms from the masticatory system in the adult population. J Oral Rehabil 2001 Sep;28(9):842-2.

9. Clarke NG, Townsend GC. Distribution of nocturnal bruxism pattern in man. J Oral Rehabil 1984 Nov;11(6):529-34.

10. Dal Fabro C. Bruxismo durante o sono e apnéia - aspectos de interesse do cirurgião-dentista [dissertação]. Bauru: Faculdade de Odontologia de Bauru, USP; 1997.

11. Gavish A, Halachmi M, Winocur E, Gazit E. Oral habits and their association with signs and symptoms of temporomandibular disorders in adolescent girls. J Oral Rehabil. 2000 Jan;27(1):22-32

12. Glaros AG. Incidence of diurnal and noturnal bruxism. J Prosthet Dent. 1981 May;45(5):545-9.

13. Grippo JO, Simring M, Schreiner S. Attrition, abrasion, corrosion and abfraction revisited: a new perspective on tooth surface lesions. J Am Dent Assoc. 2004 Aug;135(8):1109-18.

14. Hugoson A, Bergendal T, Ekfeldt A Helkimo M. Prevalence and severity of incisal and oclusal tooth wear in an adult Swedish population. Acta Odontol Scand. 1988 Oct;46(5):255-65

15. Johansson A, Haraldson T, Omar R, Kiliaridis S, Carlsson GE. A system to assessing the severity and progression of occlusal tooth wear. J Oral Rehabil. 1993 Mar;20(2):125-31

16. Kampe T, Edman G, Bader G, Tagdae T, Karlsson S. Personality traits in a group of subjects with long-standing bruxing behaviour. J Oral Rehabil. 1997 Aug;24(8):588-93
17. American academy of sleep medicine. International Classification of sleep disorders. 2 nd ed. Westchester, IL: American academy of sleep medicine; 2005

18. Lavigne GJ, Rompré PH, Montplaisir JY. Sleep bruxism: validity of clinical research diagnostic criteria in a controlled polysomnographic study. J Dent Res. 1996 Jan;75(1):546-52.

19. Macaluso GM, Guerra P, Di Giovanni G, Boselli M, Parrino L, Terzano MG. Sleep bruxism is a disorder related to periodic arousals during sleep. J Dent Res. 1998 Apr;77(4):565-73.

20. Ohayon MM, Li KK, Guilleminaut C. Risk factors for sleep bruxism in the general population. Chest 2001 Jan;119(1):53-61.

21. Rodrigues L, Lemos JBD, Tokura M, Luz JGC. Freqüência de hábitos parafuncionais e suas manifestações clínicas em pacientes com disfunções da articulação temporomandibular. Rev Odontol UNICID 2001 Maio-ago;13(2):113-23.

22. Rugh JD, Harlan J. Noturnal bruxism and temporomandibular disorders. Adv Neurol. 1988;49:329-39.

23. Saletu A, Parapatics S, Saletu B, Anderer P, Prause W, Putz H, et al. On the Pharmacotherapy of Sleep Bruxism: Placebo-Controlled Polysomnographic and Psychometric Studies with Clonazepam. Neuropsychobiology 2005 May; 51(4):214-25.

24. Seligman DA, Pullinger AG, Solberg WK. The prevalence of dental attrition and its association with factors of age, gender, occlusion, and TMJ symptomatology. J Dent Res. 1988 Oct; 67(10):1323-33.

25. Takemura T, Takahashi T, Fukuda M, Ohnuki T, Asunuma T, Masuda $\mathrm{Y}$, et al. A psychological study on patients with masticatory muscle disorder and sleep bruxism. Cranio 2006 Jul;24(3):191-6.

26. Ware JC, Rugh J.D. Destructive bruxism: sleep stage relationship. Sleep 1988 Apr;11(2):172-81.

27. Watanabe T, Ichikawa K, Clark GT. Bruxism levels and daily behaviours: 3 weeks of measurement and correlation. J Orofac Pain 2003 Winter;17(1):65-73.

Recebido em 26/11/2007 Aprovado em 09/01/2009

Correspondência: Andréa Lúcia Almeida de Carvalho Rua das Macaubas s/n Ed Boulevard I apt. 101-

Bairro: Sao Francisco São Luis-MA Brasil E-mail: andrea_alac@hotmail.com 